\title{
Coordinated Analyses of a Supernova Polycrystalline Olivine Aggregate in the CO Chondrite Dominion Range 08006
}

\author{
Laura Seifert $^{1 *}$, Pierre Haenecour ${ }^{1}$, Thomas Zega ${ }^{1,2}$ and Tarunika Ramprasad ${ }^{1,2}$ \\ 1. Lunar and Planetary Laboratory, University of Arizona, Tucson, AZ, USA. \\ 2. Materials Science and Engineering, University of Arizona, Tucson, AZ, USA. \\ * Corresponding author: 1seifert@1pl.arizona.edu
}

When a massive star falls out of hydrostatic equilibrium, its stellar core contracts and then rebounds, sending a shock wave propagating through the circumstellar envelope. Rapid nucleosynthesis is triggered by the shock wave and results in a radial explosion away from the star called a supernova (SN). Circumstellar grains condense in this ejected material, and remarkably, a portion of these solids are transported through the interstellar medium and can be found preserved in primitive materials such as meteorites. Information on the structure and chemistry of silicate and oxide grains derived from supernovae (SNe) is still severely limited [1-5] and is necessary to learn about the thermodynamic and chemical conditions in SNe ejecta. Here we discuss a coordinated SIMS-FIB-TEM study of the structure and chemistry of a polycrystalline olivine aggregate thought to be derived from a supernova in the type 3.0 CO chondrite Dominion Range (DOM) 08006.

SNe grains were identified in a petrographic thin section of DOM 08006 via NanoSIMS raster-ionimaging at Washington University in St. Louis [6]. One grain, DOM-35, was selected for detailed analysis using transmission electron microscopy (TEM). An electron transparent cross-section of DOM35 was prepared using well-established techniques [7] with the FEI Helios G3 focused-ion beam scanning-electron microscope (FIB-SEM) located at the Lunar and Planetary Laboratory (LPL). Coregistering isotopic data with secondary electron (SE) images, we placed Pt fiducial markers over the Oisotopic anomalous region of interest (ROI) to prevent over-thinning. A C strap was deposited on top of the Pt fiducials and the ROI to protect it from $\mathrm{Ga}^{+}$implantation and amorphization. The FIB section was then analysed at LPL using a Hitachi HF5000 S/TEM at $200 \mathrm{keV}$, which is equipped with SE detectors, scanning TEM (STEM)-based bright-field (BF) and dark-field (DF) imaging detectors, as well as an Oxford Instruments X-Max ${ }^{\mathrm{N}} 100 \mathrm{TLE}$ energy dispersive $\mathrm{X}$-ray spectroscopy (EDS) system with dual $100 \mathrm{~mm}^{2}$ windowless silicon-drift detectors (solid angle of $2 \mathrm{sr}$ ).

NanoSIMS analysis of DOM 08006 revealed an O-anomalous "hotspot" with enrichments in both ${ }^{18} \mathrm{O}$ and ${ }^{17} \mathrm{O}$ relative to solar system values, with ${ }^{17} \mathrm{O} /{ }^{16} \mathrm{O}=4.0 \mathrm{E}^{-4} \pm 2.0 \mathrm{E}^{-5}$ and ${ }^{18} \mathrm{O} /{ }^{16} \mathrm{O}=3.34 \mathrm{E}^{-3} \pm 7.0 \mathrm{E}^{-6}$ [6], placing it in the Group 4 field of circumstellar grains as defined by [8]. The hotspot has an oblate shape and measures roughly $235 \times 235 \mathrm{~nm}$, as confirmed by TEM data. STEM-EDS $(512 \mathrm{px} \times 512 \mathrm{px})$ maps of the overall FIB section were obtained at $200 \mathrm{keV}$ and reveal a matrix containing $\mathrm{Si}, \mathrm{O}, \mathrm{Mg}, \mathrm{Ca}$, $\mathrm{Fe}$ and large grains containing $\mathrm{Fe}$ and $\mathrm{S}$. DOM-35 contains $\mathrm{O}, \mathrm{Mg}$, and $\mathrm{Si}$, with local enrichment in Fe and $\mathrm{Ca}$ (Fig.1). Selected-area electron-diffraction (SAED) patterns were acquired across the hotspot and reveal DOM-35 to be an olivine aggregate. The left portion of the aggregate is a single crystal of stoichiometric forsterite (Fo85) and the right portion is a polycrystalline assemblage. Measurements of the polycrystalline region, together with EDS data indicate an Fe-rich olivine (Fo65).

Conditions within SNe are poorly constrained due to their highly energetic environments. It is therefore challenging to constrain the conditions of such environments via comparison of grain data with 
thermodynamic models. However, some studies offer insight, for example, [9] used model compositions of thin layers of ejecta within the main burning zones of type-II SNe, computed by [10], to construct the chemical compositions of minerals condensed by equilibrium processes in 15-, 21- and 25- solar-mass SNe. We can place constraints on the progenitor SN of DOM-35 via comparison of DOM-35 to model predictions. In comparison to [9], the ${ }^{16} \mathrm{O} /{ }^{18} \mathrm{O}$ ratio of DOM-35 is most consistent with a 15- solar-mass $\mathrm{SN}$, and the stoichiometric (Fo85) single-crystal forsterite is consistent with equilibrium condensation in a 15 to 25- solar-mass SN between 1063-1575 K. We note that Nozawa et al. [11] developed a model in which forsterite could condense in unmixed SN ejecta through non-steady-state nucleation and grain growth. However, model output by [9] demonstrated that in order to produce Fe-rich compositions of $\mathrm{X}_{\mathrm{Fa}}>0.03$, which we observe in the polycrystalline region of DOM-35 (Fo65), mixing between SN layers is required. Astronomical observations of SNe remnants support such mixing and show heterogeneous, clumpy ejecta and large-scale mixing, e.g. [12]. Thus, while forsterite condensation in an unmixed zone of the progenitor star is possible, it seems unlikely that both a single-crystal forsterite grain and Fe-rich polycrystalline olivine aggregate as seen in DOM-35 could accrete together without significant mixing [13].

\section{References:}

[1] S Messenger et al., Science 309 (2005), p. 737.

[2] C Floss et al., Geochimica et Cosmochimica Acta 70 (2006), p. 2371.

[3] T Zega et al., The Astrophysical Journal 730 (2011), p. 83.

[4] A Nguyen et al., The Astrophysical Journal 818 (2016), p. 51.

[5] A Takigawa et al., LPSC XLV Conference Proceedings (2014) abstr. \#1465.

[6] P Haenecour et al., Geochimica et Cosmochimica Acta 221 (2018), p. 379.

[7] T Zega et al., Meteoritics \& Planetary Science 42 (2007), p. 1373.

[8] L Nittler et al., The Astrophysical Journal 483 (1997), p. 475.

[9] A Fedkin et al., Geochimica et Cosmochimica Acta 74 (2010), p. 3658.

[10] T Rauscher et al., The Astrophysical Journal 576 (2002), p. 323.

[11] T Nozawa et al., The Astrophysical Journal 598 (2003), p. 785.

[12] S Abarzhi et al., Proceedings of the National Academy of Sciences (2018), \#201714502.

[13] We gratefully acknowledge the late Professor Christine Floss for her contributions to the identification of DOM-35 through NASA grants NNX14AG25G and NNX12AN77H. We also acknowledge NASA grants NNX12AL47G, NNX15AJ22G (Research support) and NSF grant 1531243 for funding instrumentation in the Kuiper Materials Imaging and Characterization Facility at LPL.

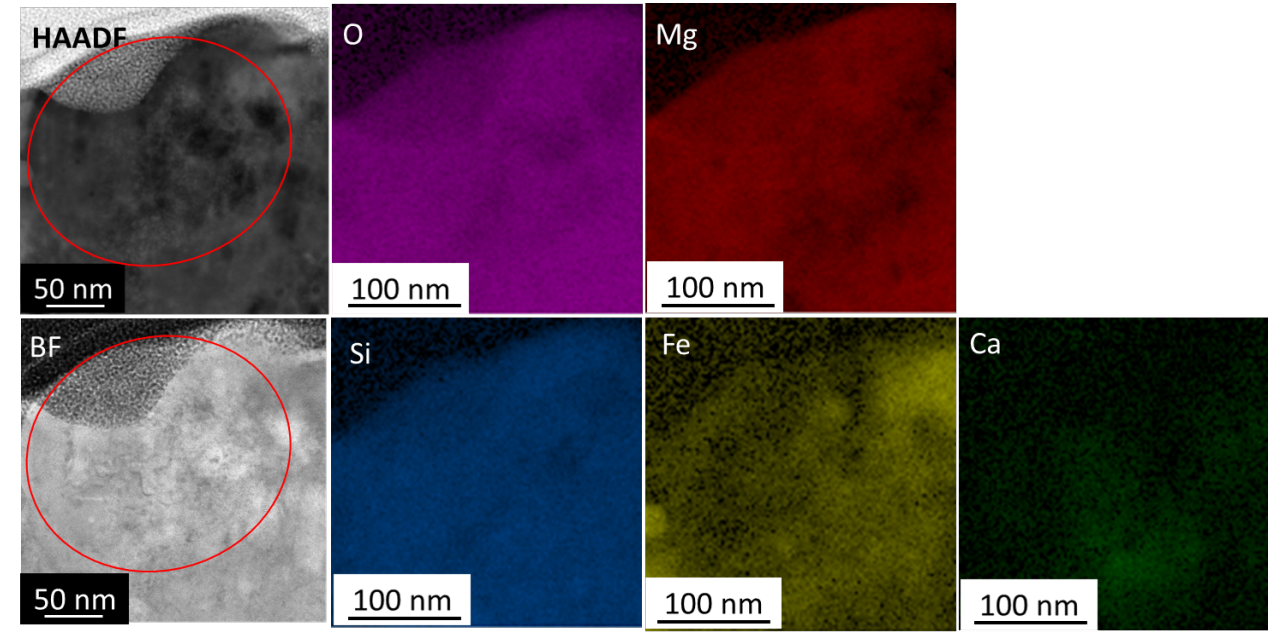

Figure 1. EDS maps of DOM-35 with HAADF and BF images for comparison. 